\title{
Effect of Exercise on the Plasma Nonesterified Fatty Acid Composition of Dogs and Goats: Species with Different Aerobic Capacities and Diets
}

\author{
Grant McClelland ${ }^{a, 1}$, Georges Zwingelstein ${ }^{b}$, C. Richard Taylor ${ }^{c}$ and Jean-Michel Weber ${ }^{a, *}$ \\ ${ }^{a}$ Biology Department, University of Ottawa, Ottawa, Ontario, K1N 6N5 Canada, Institut Michel Pacha, \\ Université de Lyon, 83500 La Seyne sur Mer, France and ${ }^{c}$ Concord Field Station, \\ Museum of Comparative Zoology, Harvard University, Cambridge, Massachusetts 02138
}

\begin{abstract}
The goals of this study were to determine: (i) whether mammals mobilize particular nonesterified fatty acids (NEFA) preferentially during locomotion, (ii) if differences in aerobic capacity or diet can affect the pattern of NEFA mobilization and (iii) which individual NEFA are most representative of total NEFA concentration changes, to use them as tracers for turnover studies. Individual NEFA were measured in trained dogs and goats $\left(\mathrm{VO}_{2} \max \operatorname{dog} / \mathrm{NO}_{2}\right.$ max goat $=2.2$; where $\mathrm{VO}_{2} \max =\max$ imal oxygen consumption) during treadmill exercise at 40 and $60 \% \mathrm{VO}_{2}$ max. Important interspecies differences in individual NEFA concentrations could be attributed to differences in aerobic capacity. The more aerobic species (dog) had much higher plasma NEFA concentrations for all but one NEFA (18:0), when compared with the low-aerobic species (goat). In addition, exercise caused a large increase in concentration of individual NEFA in the dogs, with the largest increases seen in $18: 1(150 \%$ above resting values) and $16: 0$ (60\% increase), but it had no effect in goats. Therefore, the aerobic species has a much higher ability for mobilizing and transporting NEFA in plasma than its low-aerobic counterpart. Two NEFA accounted for more than half total plasma NEFA in both species, 18:1 (about 35\% total NEFA) and $16: 0(20 \%)$. Calculation of variability in percent composition reveals that oleate and palmitate also closely reflect changes in total NEFA and are therefore the most appropriate tracers for in vivo kinetic studies in exercising mammals. Differences in diet and digestion physiology explained some differences in the plasma NEFA composition of the two species; this was reflected in the percent contributions of individual fatty acids to total NEFA: in dogs 18:1 > 16:0>18:2 > 18:0, while in goats 18:1> $16: 0>18: 0>18: 2$. Also, only goats had $18: 3$ ( $6 \%$ total NEFA), a fatty acid of plant origin, while only dogs had $16: 1$ (7\% total NEFA). Overall, however, the plasma NEFA composition of goats did not exactly reflect that of their diet due to preabsorptive modification of unsaturated fatty acids, while in dogs there was a good correlation between dietary and plasma NEFA.

Lipids 30, 147-153 (1995).
\end{abstract}

'Present address: Department of Zoology, University of British Columbia, 6270 University Blvd., Vancouver, B.C., V6T IZ4 Canada.

*To whom correspondence should be addressed at Biology Department, University of Ottawa, 30 Marie Curie, Ottawa, Ontario, KIN 6N5 Canada.

Abbreviations: ANOVA, analysis of variance; $\mathrm{CV}$, coefficient of variation; $\mathrm{GC}$, gas chromatography; NEFA, nonesterified fatty acid; $\mathrm{VO}_{2}$, rate of oxygen consumption; $\mathrm{VO}_{2}$ max, maximal rate of oxygen consumption or aerobic capacity.
Fatty acids represent a crucial source of oxidative fuel for muscle contraction, and vertebrates are known to store enough triacylglycerol to sustain exercise for several days $(1,2)$. Mammals adapted for prolonged locomotion can oxidize nonesterified fatty acids (NEFA) at much higher rates than sedentary, low-aerobic species $(3,4)$, in part because of their increased capacity to mobilize NEFA from adipose tissue (5), but also because of their higher rates of circulatory NEFA transport (4). At equivalent exercise intensities (same percentage $\mathrm{VO}_{2} \max$; where $\mathrm{VO}_{2} \max =$ maximal oxygen consumption), endurance-adapted mammals transport NEFA at much higher rates than do low-aerobic species, possibly because the plasma albumins of such species allow them to maintain a much higher total NEFA concentration in plasma (4). Major changes in total plasma concentration occur during and after exercise $(4,6)$, but it is not clear whether all individual NEFA change in parallel, or if there is preferential mobilization or use of certain fatty acids (7).

Dogs and goats were chosen as representative species of endurance-adapted and sedentary mammals for this and other studies $(3-5,8)$ because their aerobic capacities $\left(\mathrm{VO}_{2} \max \right)$ differ by a factor of 2.2. In addition, these species show major differences in diet and digestive physiology $(9,10)$. In nonruminant mammals, including dogs and humans, plasma NEFA patterns closely reflect the fatty acid composition of adipose tissue triacylglycerol (7), and the composition of stored triacylglycerol is itself closely related to dietary fatty acid patterns $(10,11)$. In contrast, ruminant species, such as the goat, show important preabsorptive modifications of NEFA. For example, a significant portion of 18 carbon polyunsaturated NEFA are hydrogenated to stearate (9). These significant differences in energy metabolism, diet and digestion suggest that dogs and goats should differ in their plasma NEFA patterns, and that exercise should have a different effect on the two species.

Preferential mobilization and oxidation of particular NEFA also influence the choice of a proper fatty acid tracer for in vivo kinetics studies of lipid fluxes in mammals, and the same individual NEFA may not be an appropriate tracer for all species. NEFA turnover rate is usually measured by 
continuous infusion of a labelled fatty acid, and this technique is based on the assumption that the individual NEFA used as a tracer is representative of the total NEFA pool (12). Traditionally, palmitate and oleate have been chosen almost exclusively because they are the most abundant in human plasma $(13,14)$. However, it is not known whether these particular NEFA can be used interchangeably in other species or if other acids would be more suitable. As a first attempt to address this issue, and because concentration and flux are usually correlated $(13,15)$, we have quantified changes in percent composition for each plasma NEFA and calculated its coefficient of variation (CV) throughout conditions of extreme fluctuations in total concentration (i.e., exercise and recovery). Theoretically, the NEFA whose percentage composition shows the lowest CV is the best representative of the total NEFA pool. The goals of this study were, therefore, to measure the concentration of individual NEFA in the plasma of exercising dogs and goats: (i) to determine whether particular NEFA are mobilized or used preferentially during locomotion, (ii) to determine whether differences in diet or aerobic capacity can have an effect on the pattern of NEFA mobilization and (iii) to find the most appropriate NEFA tracer(s) for in vivo kinetic studies by establishing which NEFA best represents the total plasma NEFA pool in the two species.

\section{MATERIALS AND METHODS}

Animals, training and experimental protocols. All experimental procedures were approved by the Animal Care Committee of Harvard University (Cambridge, MA). Five months before the experiments, three African pygmy goats (Capra hircus, two females and one male, body mass $31.0 \pm 1.3 \mathrm{~kg}$ ) and three dogs (Canis familiaris, all females, $24.9 \pm 1.8 \mathrm{~kg}$ ) were started on a training schedule using a motorized treadmill at different speeds. Animals were trained at least four times weekly until their oxygen consumption $\left(\mathrm{VO}_{2}\right)$ was reproducible at each speed. $\mathrm{VO}_{2}$ was measured with an open flow system as described by Fedak et al. (16). All training and experiments were performed at 18 and $29 \%$ incline for goats and dogs, respectively. To allow meaningful comparisons, exercise intensities were standardized according to maximal oxygen consumption ( $\mathrm{VO}_{2} \mathrm{max}$ ), and the two species were always measured at the same percentage of their $\mathrm{VO}_{2} \mathrm{max}$. The $\mathrm{VO}_{2}$ max of the individual animals used here had been measured previously (3), and each animal was specifically trained to run for $2 \mathrm{~h}$ at $40 \% \mathrm{VO}_{2} \max$ and $1 \mathrm{~h}$ at $60 \%$ $\mathrm{VO}_{2}$ max. In the present study, $60 \% \mathrm{VO}_{2} \max$ was chosen as the highest intensity because lipolysis (5) and circulatory fatty acid delivery (4) are both maximized at this work rate. Recovery was also monitored because the highest plasma fatty acid concentrations are known to occur shortly after the end of exercise in several species including dogs (4) and humans $(6,17)$

Goats were fed hay (NEFA composition: 25.5\% 16:0, $0.4 \% 16: 1,3.1 \% 18: 0,14.2 \% 18: 1,27.4 \% 18: 2$ and $26.5 \%$ $18: 3$ ) and they were housed in a large outdoor paddock with shelter. The dogs were fed dry dog chow (5\% fiber, $25 \%$ protein and $10 \%$ fat with the following composition: $24.5 \%$ of total NEFA as 16:0, 0.3\% 16:1, 14.6\% 18:0, 42.1\% 18:1 and $15.5 \% 18: 2$ ), and they were housed in a large indoor-outdoor run. Food was withheld for $18 \mathrm{~h}$ before measurements, but the animals had unlimited access to water. One day before each experiment, a PE-50 catheter was placed in the aorta via the carotid artery as described previously (5). Blood samples (2 $\mathrm{mL}$ each) were drawn 15,10 and $5 \mathrm{~min}$ before exercise, and at times $0,2,5,10,20,40,60,80,100$ and 120 min during exercise at $40 \% \mathrm{VO}_{2} \max$. At $60 \% \mathrm{VO}_{2} \max$, samples were taken at times $0,2,5,10,20,30,40,50$ and $60 \mathrm{~min}$. Additional samples were drawn 2, 5, 10, 20,30,40, 50 and $60 \mathrm{~min}$ after exercise had ended (recovery period). Samples were spun, and the plasma was separated and frozen immediately. The two exercise intensities were measured in different experiments, and two experiments on the same animal were separated by at least one week.

NEFA concentrations. Individual NEFA concentrations were measured on a Hewlett-Packard 5890 series II gas chromatograph (Mississauga, Canada) equipped with an automatic injection system (HP 7673) and flame-ionization detector. NEFA extraction and methylation were performed with the method of Tserng et al. (18) modified by increasing methylation time to $20 \mathrm{~min}$. Preliminary thin-layer chromatography (TLC) and gas chromatography (GC) analyses using plasma and standard polyunsaturated fatty acids, in-

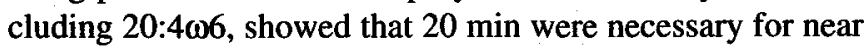
complete methylation. One-hundred $\mu \mathrm{L}$ of plasma were methylated with $1 \mathrm{~mL}$ 2,2-dimethoxypropane (Sigma, St. Louis, MO) after adding $20 \mu \mathrm{L}$ of conc. $\mathrm{HCl}$. The reaction was stopped after $20 \mathrm{~min}$ by the addition of $10 \mu \mathrm{L}$ pyridine, and the NEFA methyl esters were recovered by two extractions with $750 \mu \mathrm{L}$ isooctane $(\mathrm{BDH}$, Toronto, Ontario, Canada). Analyses were performed on a $30-\mathrm{m}$ fused silica capillary column (DB-23; J\&W Scientific, Folsom, CA) kept at $170^{\circ} \mathrm{C}$ for $35 \mathrm{~min}$ after injection, raised to $220^{\circ} \mathrm{C}$ at a rate of $50^{\circ} \mathrm{C} / \mathrm{min}$, and kept at that temperature up to $46 \mathrm{~min}$. The injection port temperature was $210^{\circ} \mathrm{C}$ and the detector was kept at $250^{\circ} \mathrm{C}$. NEFA standards (Supelco, Bellefonte, PA) were injected for GC calibration, and heptadecanoic acid (12 $\mu \mathrm{g} / 100 \mu \mathrm{L}$, in methanol) was used as an internal standard. This internal standard was added to plasma before methylation, and recovery ranged between 95 and $98 \%$.

Statistical analysis. Results were analyzed with one- and two-way analyses of variance (ANOVA) after percentages were arcsine transformed. In cases where the data failed tests for normality (Kolmogorov-Smirnov), a nonparametric Kruskal-Wallis one-way ANOVA on ranks was used. Bonferroni's $t$-test for multiple comparisons was used to compare mean NEFA concentrations during exercise and recovery with those at rest, and to compare the different phases in Table 1. A Dunn's multiple comparison was performed when sample sizes were unequal. Linear regressions were performed on all exercise values for each species separately to see if NEFA concentrations changed significantly over time. 
TABLE 1

Fatty Acid Composition ${ }^{a}$ (wt \% total fatty acids) in Plasma of Dogs and Goats Before, During and After Exercise at Two Intensities ${ }^{b}$

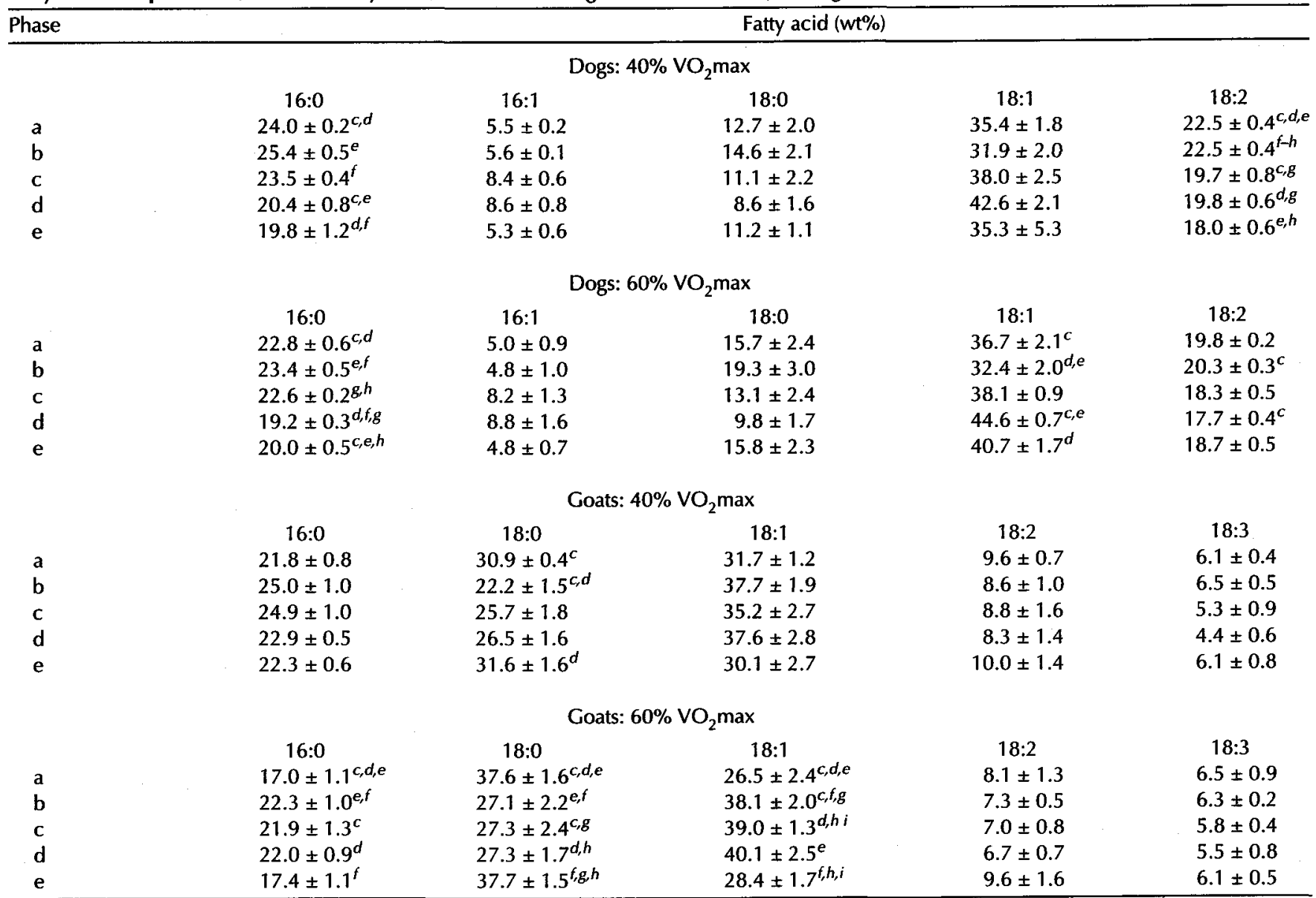

Values are averages of two determinations in each of three animals.

${ }^{b}$ See Figure 2 and Results section for definition of phases a through e. Animals were exercised at 40 and $60 \% \mathrm{VO}_{2} \max \left(\mathrm{VO}_{2} \max =\operatorname{maximal}\right.$ oxygen consumption).

${ }_{c-i}$ Within each column and each treatment, means for phases sharing the same superscript are significantly different from each other at $P<0.05$.

CVs (19) were calculated separately in each species for each NEFA throughout exercise and recovery. Data for the two exercise intensities were combined to calculate these coefficients. All values presented are means and their standard errors (SEM).

\section{RESULTS}

NEFA concentrations. Plasma concentrations of palmitic, oleic and linoleic acids were always much higher in dogs than in goats before, during and after exercise at 40 and $60 \%$ $\mathrm{VO}_{2} \max (P<0.001$, Fig. 1). Stearic acid concentration was not different between the two species $(263 \pm 6 \mathrm{nmoles} / \mathrm{mL})$ and remained at resting levels throughout exercise at both intensities and during recovery. In dogs, with the exception of stearate, the concentration of all NEFA showed a steady increase during exercise at both intensities (linear regressions, $P<0.01$ ), and an overshoot in concentration occurred after the end of work (Fig. 1). NEFA concentrations were maximal during the overshoot when they reached levels more than twice resting values shortly after exercise ended $(P<0.05)$.
Dog 16:0 reached maximal concentrations of $690 \pm 47$ and $649 \pm 78 \mathrm{nmol} / \mathrm{mL}$ shortly after exercise at 40 and $60 \%$ $\mathrm{VO}_{2} \max$, respectively. In the same species, 18:1 concentration increased throughout exercise and reached maximum values of $1495 \pm 153$ and $1479 \pm 109 \mathrm{nmol} / \mathrm{mL}, 20 \mathrm{~min}$ after ending exercise at 40 and $60 \% \mathrm{VO}_{2} \max$, respectively. Recovery overshoot values for $18: 2$ were $678 \pm 23 \mathrm{nmol} / \mathrm{mL}$ ( 20 min after the $40 \% \mathrm{VO}_{2} \max$ run) and $614 \pm 76 \mathrm{nmol} / \mathrm{mL}(10$ min after the $60 \% \mathrm{VO}_{2} \max$ run) (Fig. 1). In sharp contrast to the response observed in dogs, the NEFA concentrations of goats never increased above resting values $(P>0.05)$. Palmitic acid remained at an average level of $153 \pm 8$ $\mathrm{nmol} / \mathrm{mL}$, oleic àcid stayed at $227 \pm 13$ and linoleic acid at 66 \pm 5 for both, 40 and $60 \% \mathrm{VO}_{2} \max$ runs $(P>0.05$, Fig. 1$)$. Within each species, the overall patterns of changes in NEFA concentration were similar at the two exercise intensities.

NEFA composition. Palmitic, stearic, oleic and linoleic acids were present in both species. Significant amounts of 16:1 were only found in dog plasma (7\% of total NEFA), and $18: 3$ was present only in goat plasma $(6 \%)$. Changes in the distribution of individual NEFA over time are presented in 


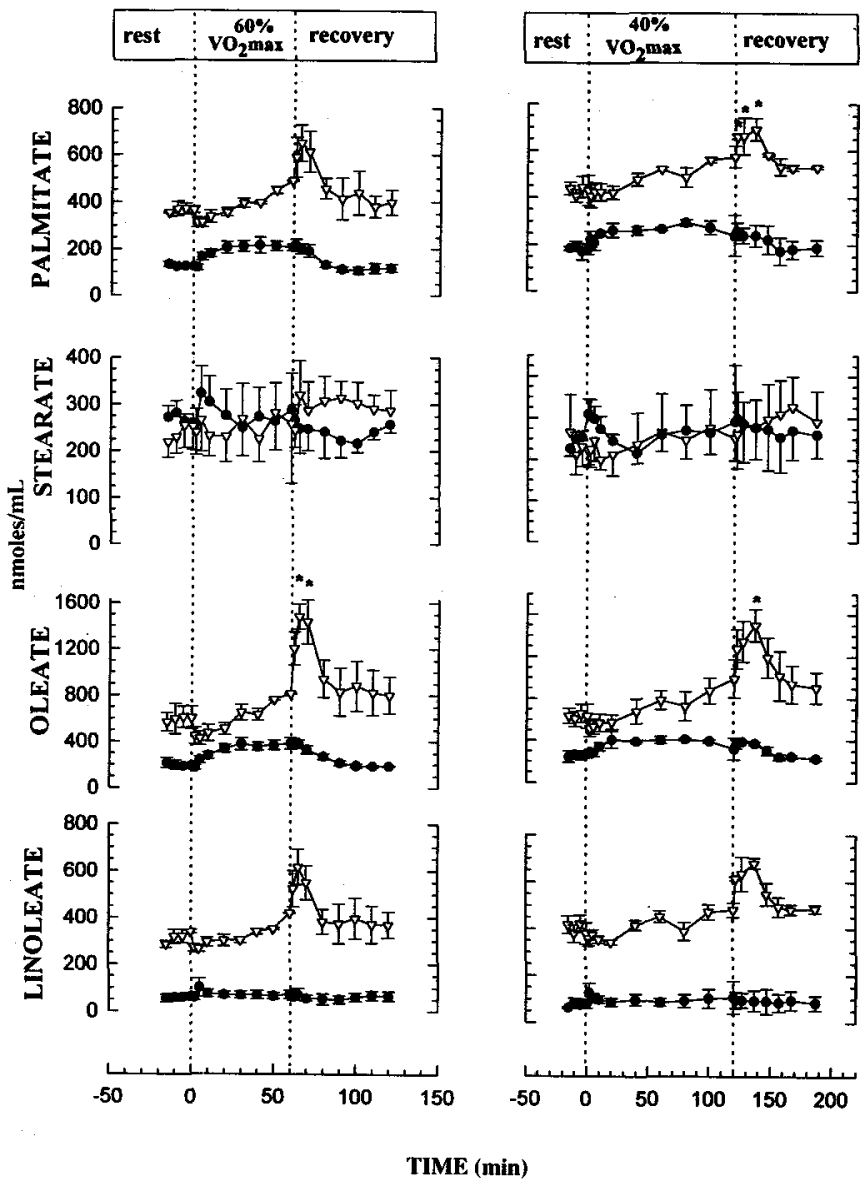

FIG. 1. Mean concentrations of individual nonesterified fatty acid in the plasma of $\operatorname{dogs}(\nabla, n=3)$ and African pygmy goats $(0, n=3)$ before, during and after a $1-\mathrm{h}$ run at $60 \%$ maximal oxygen consumption $\left(\mathrm{VO}_{2}\right.$ max) and a 2 -h run at $40 \% \mathrm{VO}_{2}$ max.

Table 1 as percentages of total NEFA concentration. Experimental protocols were divided in five phases to facilitate comparison: Phase a was the preexercise control period. Phase $b$ was the onset of exercise (average value between 20 and 40 min of exercise at $40 \% \mathrm{VO}_{2} \max$, and between 20 and $30 \mathrm{~min}$ at $60 \% \mathrm{VO}_{2} \max$ ). Phase $\mathrm{c}$ was the end of exercise (average value between 100 and $120 \mathrm{~min}$ at $40 \% \mathrm{VO}_{2} \max$, and between 50 and $60 \mathrm{~min}$ at $\left.60 \% \mathrm{VO}_{2} \max \right)$. Phase $\mathrm{d}$ was the beginning of recovery (127 to $137 \mathrm{~min}$ at $40 \% \mathrm{VO}_{2}$ max and 65 to $70 \mathrm{~min}$ at $\left.60 \% \mathrm{VO}_{2} \max \right)$. Finally, phase e was the end of recovery (167 to $187 \mathrm{~min}$ at $40 \% \mathrm{VO}_{2} \mathrm{max}$ and 110 to $120 \mathrm{~min}$ at $60 \% \mathrm{VO}_{2} \mathrm{max}$ ). The five phases are illustrated in Figure 2, and significant differences between phases are indicated by superscripts in Table 1. Oleic acid was the most abundant NEFA, representing on average over $35 \%$ of total NEFA in both species. Similarly, the relative importance of 16:0 was the same in dogs and goats; averaged throughout exercise and recovery at both intensities, $16: 0$ accounted for $21 \%$ of total NEFA concentration. Together, therefore, 18:1 and 16:0 represented more than half of total NEFA in both species. At $40 \% \mathrm{VO}_{2} \mathrm{max}$, the percentages of 18:1 and 16:0 were not different between species $(P>0.05)$. However, at $60 \% \mathrm{VO}_{2} \max$, the percentages of $18: 1$ and $16: 0$ were both significantly

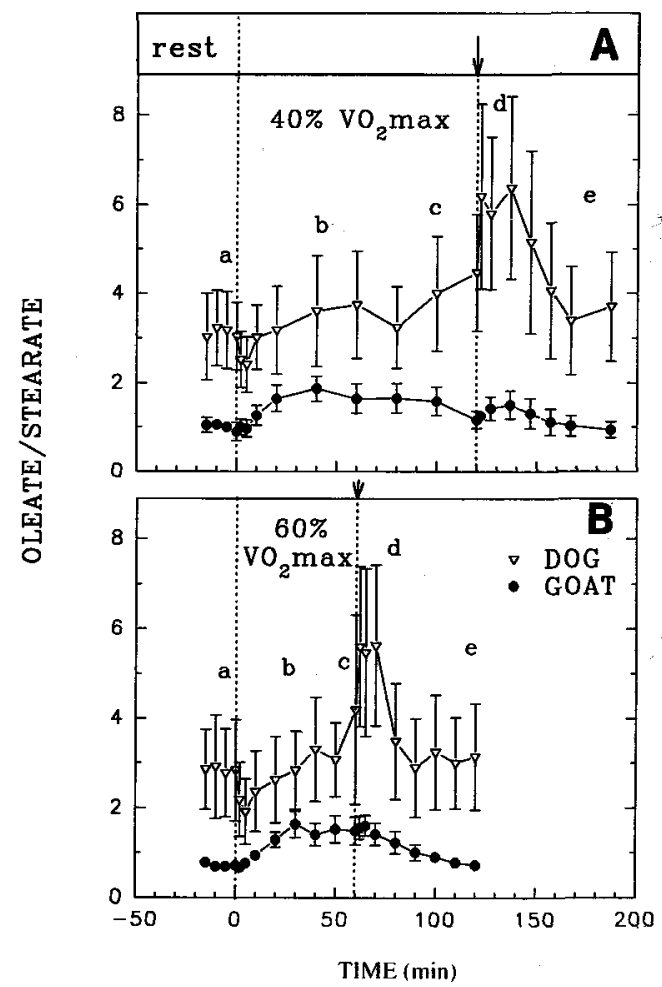

FIG. 2. Ratio of oleic to stearic acid concentration in the plasma of dogs $(\nabla, \mathrm{n}=3)$ and goats $(, \mathrm{n}=3)$, before, during and after exercise (A) a 2 -h run at $40 \% \mathrm{VO}_{2} \max$ and $(\mathrm{B})$ a 1 -h run at $60 \% \mathrm{VO}_{2}$ max. Arrows indicate when exercise was terminated. See Figure 1 for abbreviation.

higher in dogs than in goats $(P<0.01)$ (39 vs. $34 \%$ for $18: 1$, and 22 vs. $20 \%$ for 16:0). The percentage of 18:0 was almost two times higher in goats than in dogs (goats: 24 and $27 \%$ vs. dogs: 13 and $16 \%$ at 40 and $60 \% \mathrm{VO}_{2}$ max, respectively). The situation was reversed for 18:2 because this NEFA accounted for $20 \%$ in dogs, but only $9 \%$ in goats.

To emphasize important interspecies differences in the effect of exercise on plasma NEFA composition, the concentration ratio of 18:1 to $18: 0$ was calculated for both species (Fig. 2). This ratio was always much higher in dogs than in goats $(P<0.001)$. The difference between the two species varied during and after exercise at both intensities, but it was most pronounced early in recovery. At rest, the ratio was more than 2-fold higher in dogs than in goats (over $2.5 \mathrm{vs}$. $0.9)$. The ratio in goats doubled during exercise before returning progressively to resting levels during the recovery period. In dogs, the ratio increased slowly during exercise before a much sharper rise in the first 20 min of recovery. High maximal values of 5.4 and 5.0 were reached after ending the runs at 40 and $60 \% \mathrm{VO}_{2} \max$, respectively (Fig. 2).

CVs for individual NEFA composition. Mean CVs for all exercise and recovery values at both 40 and $60 \% \mathrm{VO}_{2} \max$ were calculated for the percent composition of each NEFA (Table 2). Palmitic and oleic acids had the lowest and second lowest $\mathrm{CV}$ in both species, respectively. The variability in composition of all the other NEFA was clearly higher than for 
TABLE 2

Mean Coefficients of Variation for Individual Fatty Acid Composition (\% total nonesterified fatty acid concentration) Throughout Exercise and Recovery in Trained Goats and Dogs ${ }^{a}$

\begin{tabular}{lcc}
\hline & Goat & Dog \\
\hline $16: 0$ & 9.06 & 8.86 \\
$\mathbf{1 6 : 1}$ & $-b$ & 22.21 \\
$\mathbf{1 8 : 0}$ & 13.07 & 19.11 \\
$\mathbf{1 8 : 1}$ & 12.30 & 10.09 \\
$\mathbf{1 8 : 2}$ & 16.70 & 16.43 \\
$18: 3$ & 15.63 & $-b$ \\
\hline
\end{tabular}

Values for two exercise intensities were combined (40 and $60 \%$ maximal rate of oxygen consumption or aerobic capacity). ${ }^{b}$ Not detected.

16:0 or 18:1, and the highest CVs were observed for $16: 1$ in dogs and 18:2 in goats.

\section{DISCUSSION}

The present study shows that exercise has a very different effect on the concentration of individual plasma NEFA in sedentary animals, such as goats, than in highly aerobic, endurance-adapted animals, such as dogs. It also reveals that important differences in plasma NEFA composition between these two species can be attributed to dietary NEFA and differences in digestion. Moreover, variability in percent composition for individual NEFA shows that, as in humans, changes in plasma 16:0 and 18:1 most closely reflect changes in total NEFA concentration of dogs and goats, suggesting that these two fatty acids are most appropriate as tracers for NEFA kinetic studies in mammals in general.

Except for stearic acid, plasma NEFA concentrations were much higher in dog than in goat (Fig. 1), allowing the species with high aerobic capacity (dog) to deliver this important oxidative fuel to working muscles at much higher rates than the sedentary species with low aerobic capacity (goat). The high concentrations found in dogs may be possible because this species possesses a plasma albumin with higher NEFA binding capacity than goat albumin. We have recently suggested this higher albumin binding capacity for fatty acids in dogs as an adaptation for endurance locomotion (4).

Another major difference between the two species lies in the fact that exercise causes a large increase in NEFA concentration in dogs (except for 18:0), but has no effect in goats, where it stays at resting levels throughout work and recovery (Fig. 1). Humans have a similar response to that described here for dogs: except for 18:0, their plasma NEFA concentration increases by $25 \%$ after exercising at moderate intensity for $30 \mathrm{~min}$ (21). These results clearly show that dogs and humans mobilize NEFA reserves during exercise, because, in these species, concentrations of individual NEFA are known to be well correlated with rates of turnover $(13,15)$ and oxidation $(15,20)$. Therefore, in dogs and humans, increases in concentration can be used as an index of NEFA mobilization. In contrast, the lack of change in plasma NEFA concentration of exercising goats reveals that they are able to increase NEFA flux while keeping plasma concentration constant. In goats, NEFA mobilization and oxidation are kept closely balanced throughout exercise and recovery. This observation shows that concentration and turnover of 16:0 and 18:1 are not correlated in goats because they do increase oxidation during exercise, even though lipid utilization is not stimulated as much as in dogs (4).

In dogs, 18:1 and 16:0 have the highest concentrations at rest and show the greatest relative increase during the onset of exercise. These results indicate that dogs mobilize these two NEFA preferentially compared with other NEFA, and probably at much higher rates than do goats. However, these conclusions must remain tentative because percent extraction in locomotory muscles may change with exercise and between species. For example, stearic acid does not follow the typical pattern observed for all other NEFA, and it does not appear to contribute to the overall increase in oxidative fuel use associated with exercise in mammals studied to date. It has been proposed that the fractional uptake of 18:0 may increase in exercising humans (21), suggesting that rates of release from adipose tissue and uptake by working muscles may both increase in parallel and stay exactly matched during physical work (17). This would make stearic acid flux independent of its plasma concentration, and an increase in 18:0 mobilization/oxidation would only be discernible by flux measurements. Stearic acid kinetics should be investigated thoroughly in exercising mammals to determine whether the scenario proposed above actually occurs. In goats, the absence of NEFA mobilization throughout exercise may be the consequence of high plasma glucose availability. Recent experiments on the same animals reveal that glucose concentration increases progressively during exercise in goats, but is very steady in dogs (Weber, Roberts and Taylor, unpublished results). Hyperglycemia can decrease fatty acid turnover by as much as $30 \%$ in humans (22), and glucose could therefore be an important inhibitor of lipid mobilization in exercising goats.

Dogs (Fig. 1) and humans $(6,17)$ show a very large postexercise increase in NEFA concentration. Fifteen min after the $40 \% \mathrm{VO}_{2} \max$ run, dog 16:0 and 18:1 concentrations reached maximal values 60 and $150 \%$ above resting levels, respectively. In striking contrast, no change was observed in recovering goats. The large concentration overshoots seen in dogs and humans indicate that both these species, unlike goats, probably lack rapid control mechanisms for lipolysis $(5,6,17)$. High plasma NEFA concentrations after exercise may play a role in the regulation of fuel oxidation by inhibiting muscle glycolysis through the glucose-fatty acid cycle, and by promoting muscle glycogen resynthesis through an increase in glucose availability (17). In goats, the absence of NEFA overshoot is consistent with the fact that their lipolytic rate returns to resting levels immediately and rapidly after the end of exercise (5).

Plasma NEFA compositions of the two species investigated can be explained by differences in diet, incorporation of individual NEFA in triacylglycerols, and in situ modifications of NEFA in adipose tissue and liver triacylglycerol reserves. The composition of NEFA in plasma is known to reflect differen- 
tial NEFA incorporation into adipose tissue triacylglycerol, and it has been reported that under conditions of enhanced lipolysis, the composition of plasma NEFA approaches that of adipose tissue (7). In vivo dog experiments and in vitro measurements on rat epididymal fat pads show that the rate of release of each individual NEFA is correlated with its relative concentration in adipose tissue (7). For nonruminants, both adipose tissue triacylglycerol and plasma NEFA reflect dietary composition (11) due to little preabsorptive modification (10). Indeed, the composition of plasma NEFA in dogs (Table 1) closely reflects that of their diet (see Materials and Methods section). In ruminant mammals, NEFA composition of adipose tissue triacylglycerol may also be influenced by postabsorptive modifications of dietary NEFA. For example, the much higher proportion of $18: 1$ found in goat plasma (Table 1) than in their diet may be the consequence of high activities for the enzymes fatty acid elongase and stearoyl-CoA desaturase in adipose tissue $(23,24)$.

Stearic acid represents a greater proportion of total dietary NEFA in dogs than in goats, but the opposite is true for total plasma NEFA (Table 1). This discrepancy can be accounted for by differences in digestive physiology between ruminants and nonruminants. In ruminants, dietary unsaturated fatty acids are hydrogenated in the rumen, and little polyunsaturated NEFA reach absorption sites in the small intestine $(9,25)$. For example, higher than expected levels of stearic acid are probably found in plasma because a large proportion of dietary 18 carbon polyunsaturated NEFA is hydrogenated to 18:0 prior to absorption (9). Given that goats and other ruminants absorb lower amounts of polyunsaturated NEFA than predicted from their diet (25), availability from digestion rather than differential rate of esterification between NEFA in adipose tissue triacylglycerol likely dictates the composition of adipose tissue and ultimately plasma (23).

Figure 2 shows that the concentration ratio of 18:0/18:1 is very different between dogs and goats, before, during and after exercise. This observation may be partly explained by interspecific differences in the activities of enzymes responsible for NEFA incorporation in adipose tissue triacylglycerol; lower incorporation of particular NEFA may result in their lower contribution to total plasma NEFA. Except for stearic acid, rates of incorporation in adipose tissue increase linearly with plasma concentration (23). In experiments by Lin et al. (23), 18:0 had the lowest rate of esterification in bovine adipose tissue while 16:0 had the highest. Incorporation of 18:0 in adipose tissue triacylglycerol may differ between dogs and goats due to discrepancies in the activities of fatty acyl-CoA synthetase. Low incorporation of stearic acid into bovine adipose tissue is thought to be caused by fatty acyl-CoA synthetase's low affinity for 18:0 (23). As in dogs, 18:0 does not contribute to the increase in total plasma NEFA of humans, and the 18:1/18:0 ratio increases from 3.3 at rest (compared to 2.7 in dogs) to a maximum of 4.5 (5.4 in dogs) after $50 \mathrm{~min}$ of exercise (21).

Additional differences between dogs and goats may be more directly attributed to differences in diet, and the compo- sition of plasma NEFA found here in goats is comparable with values reported previously (25). Only goat plasma contained 18:3, a NEFA of plant origin especially abundant in grass lipids $(25,26)$, and represented a relatively large proportion of total FA in the goat diet of our study (see Materials and Methods section). Palmitoleic acid was only found in dog plasma, although this NEFA has also been reported to be present in small amounts in goat plasma (25). We did not detect it in goats with the methods used in the present study.

Palmitic and oleic acids have the lowest variability in percent composition throughout exercise and recovery (Table 2). Therefore, changes in their concentrations are the most representative of changes in total plasma NEFA, and they should be the preferred labeled fatty acids for flux measurements in dogs and goats. Also, both of these acids are relatively abundant, making the measurement of their specific radioactivity or enrichment easier than with other much less concentrated NEFA. Palmitic and oleic acids have been used extensively in NEFA kinetics studies in humans $(12,13,27)$, and the present study suggests that they are also suitable for such studies in other mammalian species, even during exercise.

\section{ACKNOWLEDGMENTS}

This work was supported by an NSERC grant (Canada) to J.-M.W. (OGP0105639) and an NSF grant to C.R.T. (DCB8918371). G. McC. was the recipient of a fellowship from the French Ministry of External Affairs (CIES).

\section{REFERENCES}

1. Newsholme, E.A. (1988) in Principles of Exercise Biochemistry (Poortmans, J.R., ed.) pp. 40-77, Karger, Basel.

2. Hochachka, P.W., and Somero, G.N. (1984) Biochemical Adaptation, pp. 85-144, Princeton University Press, Princeton.

3. Roberts, T.J., Weber, J.-M., and Taylor, C.R. (1990) The Physiologist 33, A110.

4. McClelland, G., Zwingelstein, G., Taylor, C.R., and Weber, J.-M. (1994) Am. J. Physiol. 266, R1280-R1286.

5. Weber, J.-M., Roberts, T.J., and Taylor, C.R. (1993) Am. J. Physiol. 264, R797-R803.

6. Wolfe, R.R., Klein, S., Carraro, F., and Weber, J.-M. (1990) Am. J. Physiol. 258, E382-E389.

7. Nakamura, H., Faludi, G., and Spitzer, J.J. (1967) Diabetes 16, 175-180.

8. Taylor, C.R., Karas, R.H., Weibel, E.R., and Hoppeler, H. (1987) Resp. Physiol. 69, 1-27.

9. Dziuk, H.E. (1984) in Dukes' Physiology of Domestic Animals (Swenson, M.J., ed.) pp. 320-339, Cornell University Press, Ithaca.

10. Argenzio, R.A. (1984) in Dukes' Physiology of Domestic Animals (Swenson, M.J., ed.) pp. 301-310, Cornell University Press, Ithaca.

11. Jéquier, E. (1992) in Energy Metabolism: Tissue Determinants and Cellular Corollaries (Kinney, J.M., and Tucker, H.N., eds.) pp. 123-139, Raven Press, New York.

12. Spitzer, J.J. (1975) Fed. Proc. 34, 2242-2245.

13. Hagenfeldt, L. (1975) Fed. Proc. 34, 2236-2240.

14. Fredrickson, D.S., and Gordon, R.S. (1958) J. Clin. Invest. 37, 1507-1515.

15. Paul, P., and Issekutz, J.B. (1967) J. Appl. Physiol. 22, 615-622. 
16. Fedak, M.A., Rome, L., and Seeherman, H.J. (1981) J. Appl. Physiol. 51, 772-776.

17. Bahr, R., Hostmark, A.T., Newsholme, E.A., Gronnerod, O., and Sejersted, O.M. (1991) Acta Physiol. Scand. 143, 105-115.

18. Tserng, K.-Y., Kliegman, R.M., Miettinen, E.-L., and Kalham, S.C. (1981) J. Lipid Res. 22, 852-858.

19. Zar, J.H. (1984) Biostatistical Analysis, 2nd edn., p. 32, Prentice-Hall, Englewood Cliffs.

20. Havel, R.J., Carlson, L.A., Ekeland, L.G., and Holmgren, A. (1964) J. Appl. Physiol. 19, 613-618.

21. Hagenfeldt, L., Wahren, J., Pernow, B., and Ekestrom, S. (1972) J. Clin. Invest. 51, 3061-3071.

22. Carlson, M.G., Snead, W.L., Hill, J.O., Nurjhan, N., and Campbell, P.J. (1991) Am. J. Physiol. 261, E815-E820.
23. Lin, K.-C., Cross, H.R., and Smith, S.B. (1992) Lipids 27, 111-116.

24. Payne, E., and Masters, C.J. (1971) Int. J. Biochem. 2, 623-643.

25. Leat, W.M.F., and Baker, J. (1970) Comp. Biochem. Physiol. 36, 153-161.

26. Goodwin, T.W., and Mercer, E.I. (1983) Introduction to Plant Biochemistry, 2nd edn., p. 275, Pergamon Press Ltd., New York.

27. Wolfe, R.R. (1984) Tracers in Metabolic Research. Radioisotope and Stable Isotope/Mass Spectrometry Methods, p. 287, Alan R. Liss, New York.

[Received December 9, 1993, and in revised form December 16, 1994; Revision accepted December 28, 1994] 